\title{
Chemically treated multi-walled carbon nanotubes for sensor applications
}

\author{
Khurshed A. Shah \\ Nanomaterials Research Laboratory, Department of Physics, Govt. Degree College for Women, \\ Anantnag (J\&K), India \\ drkhursheda@gmail.com
}

PACS 81.07.De, 81.16.Be, 87.64.kp

DOI 10.17586/2220-8054-2016-7-3-492-493

\begin{abstract}
In this study, Multi-Walled Carbon Nanotubes (MWCNTs) were chemically treated in order to investigate the structural and chemical changes in them and to use them for sensor applications. Raman spectroscopic analysis reveals that the chemically treated MWCNTs are useful for chemical and gas sensor applications.
\end{abstract}

Keywords: Carbon nanotubes, chemical treatment, Raman spectroscopy, sensor applications.

Received: 23 January 2016

\section{Introduction}

The last decade has witnessed vigorous research activity in the field of Carbon nanotubes (CNTs) due to their extraordinary properties. There are various structures of carbon nanotubes (CNTs) depending on how the tube is rolled up and it is defined by the chiral index $(n, m)$ and its quantum properties depend on the diameter and chirality [1,2]. It exists both in single and multi-walled form. Isolated single-wall carbon nanotubes do not normally occur. Mostly, group of SWNTs led to formation of bundles of tubes, so-called nanoropes containing between 20 to hundred individual tubes $[3,4]$. There are various applications of CNTs which includes sensors as well $[5,6]$.

The CNTs as produced by the various synthesis techniques contain various impurities [7] and for many applications, these impurities have to be separated from the carbon nanotubes before they can be used for sensor applications. Purification techniques include air oxidation, acid treatment, annealing, micro filtration, sonication, ferromagnetic separation, functionalization, and chromatography techniques have been devised in order to improve the quality and yield of carbon nanotubes [8]. Among these methods in this study the chemical method has been chosen in order to attach functional groups to CNTs. The Raman spectra analysis shows that carboxylic groups have been successfully attached to the carbon nanotubes after chemical treatment. These carboxylic groups are very useful for chemical and gas sensing applications.

\section{Experimental details}

As-produced multiwall carbon nanotubes (MWCNTs) were treated in a mixture of $\mathrm{H}_{2} \mathrm{SO}_{4}, \mathrm{HNO}_{3}$ under a refluxing condenser with magnetic stir for $5 \mathrm{hr}$. After refluxing, the mixture was cooled to room temperature, diluted with methanol and filtered through Whatman filter paper. The dried sample was then analyzed by a Raman Spectrometer.

\subsection{Results and discussion}

Figure 1 shows Raman spectra of treated CNTs excited with the $532.8 \mathrm{~nm}$ laser line.

In the case of CNTs, defect induced band ( $\mathrm{D}$ band) and graphitic band (G band) are usually found in the range of $1332-1365 \mathrm{~cm}^{-1}$ and $1516-1585 \mathrm{~cm}^{-1}$ respectively. From Fig. 1, it is seen that the two peaks were observed at $1356 \mathrm{~cm}^{-1}$ (D band) and at $1577 \mathrm{~cm}^{-1}$ ( $\mathrm{G}$ band) showing the characteristics of CNTs, when the acid treatment of CNTs was conducted. Furthermore it is seen that the peak position does not change, indicating that the acid treatment does not destroy the structure of CNTs [9], although the spectra is not shown in the Fig. 1. The intensity ratio of D-to G-mode $\left(I_{D} / I_{G}\right)$ values of chemically treated and as-grown MWCNTs increases. As observed from Raman spectra, the ratio between the intensity of the $\mathrm{D}$ band and the $\mathrm{G}$ band is 0.68 . The ratio between the intensity of $G^{\prime}$ band to $G$ band is 0.43 . These results are generally attributed to the presence of more structural defects [10]. The adsorption of functional groups increases the number of defects in the structure of nanotubes, increasing the ratio $I_{D} / I_{G}$ and providing for early decomposition of CNTs. These results indicate certain insertion of defects and/or break on the structure of nanotubes and attach some functional groups which are useful for chemical and gas sensor application as many researchers have studied [11]. 


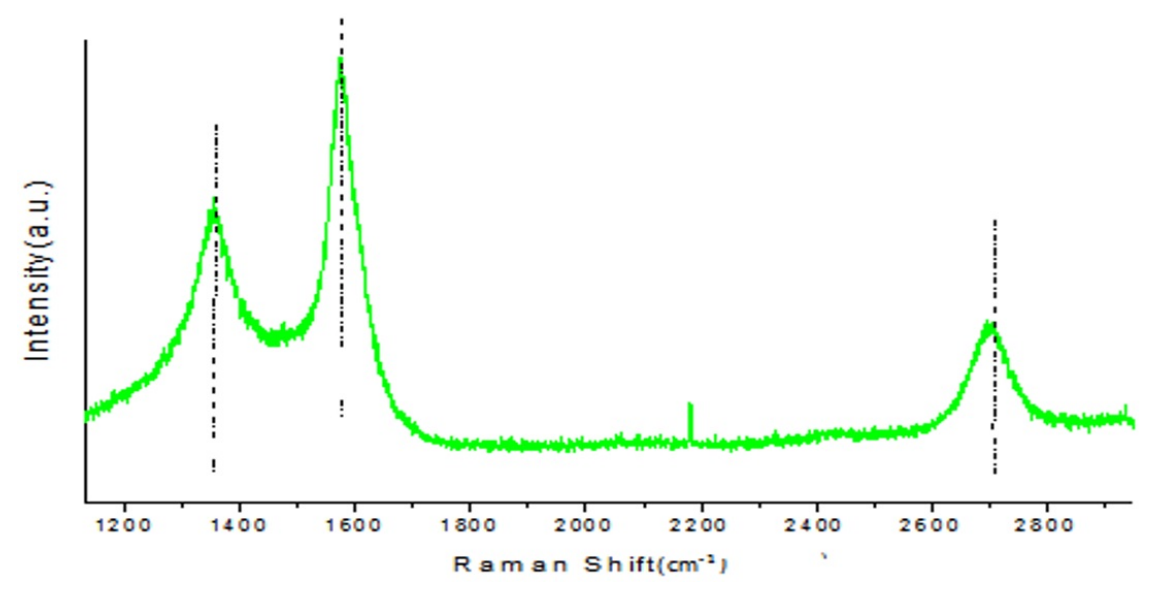

FIG. 1. Raman spectra of chemically treated carbon nanotube sample with excitation wavelength $532.8 \mathrm{~nm}$

\section{Conclusion}

The as-produced MWCNT sample was characterized by Raman spectroscopy after chemical functionalization. The analysis of Raman spectra indicated there was modification of the CNTs structure after chemical treatment resulting in an increase in the number of defect sites. Some carboxylic groups are attached with these defect sites which are very useful for chemical and gas sensing applications.

\section{Acknowledgement}

This work has been financially supported by the Department of Science and Technology, Science and Engineering Research Board (DST-SERB), Govt. of India (Project grant No.SB/S2/CMP-36/2013).

\section{References}

[1] Ebbesen T.W. Carbon Nanotubes: Preparation and Properties. CRC Press, Boca Raton, Florida, 1997.

[2] Rao A.M., Richter E., Bandow S., Chase B., Eklund P.C., Williams K.A., Fang S., Subbaswamy K.R., Menon M., Thess A., Smalley R.E., Dresselhaus G., Dresselhaus M.S. Diameter-Selective Raman Scattering from Vibrational Modes in Carbon Nanotubes. Science, 1997, 275, P. 187-191.

[3] Iijima S., Ichihashi T. Single-Shell Carbon Nanotubes of 1-nm Diameter. Nature, 1993, 363, P. $603-605$.

[4] Journet C., Bernier P. Production of Carbon Nanotubes. Appl. Phys. A, 1998, 67, P. 1-9.

[5] Liu J.H., Liu J.Y., Yang L.B., Chen X., Zhang M.Y., Meng F.L., Luo T., Li M.Q. Nanomaterial-Assisted Signal Enhancement of Hybridization for DNA Biosensors: A Review. Sensors, 2009, 9, P. 7343-7346.

[6] Balasubramanian K., Burghard M. Biosensors Based on Carbon Nanotubes. Anal. Bioanal. Chem., 2006, 385, P. $452-468$.

[7] Koshio A., Shiraishi M., Kobayashi Y., Ishihara M., Koga Y., Bandow S., Iijima S., Kokai F. Modification of Carbon Nanotubes by Laser Ablation of Copper. Chemical Physics Letters, 2004, 396, P. 410-414.

[8] Agboola A.E. et al. Conceptual Design of Carbon Nanotube Processes. Springer-Verlag, 2007, 9, P. $289-311$.

[9] Osorio A.G., Silveira I.C.L., Bueno V. L., Bergmann C.P. $\mathrm{H}_{2} \mathrm{SO}_{4} / \mathrm{HNO}_{3} / \mathrm{HCl}$-Functionalization and Its Effect on Dispersion of Carbon Nanotubes in Aqueous Media. Applied Surface Science, 2008. 255, P. 2485-2489.

[10] Tian Z.Q., Jiang S.P., Liang Y.M., Shen P.K. Synthesis and Characterization of Platinum Catalysts on Multiwalled Carbon Nanotubes by Intermittent Microwave Irradiation for Fuel Cell Applications. J. Phys Chem. B, 2006, 110, P. 5343-5350.

[11] Bekyarova E., Davis M., Burch T., Itkis M.E., Zhao B., Sunshine S., Haddon R.C. Chemically Functionalized Single Walled Carbon Nanotubes as Ammonia Sensors. J. Phys. Chem. B, 2004, 108, P. 19717-19720. 